\title{
Oral Chemotherapy Program Improves Adherence and Reduces Medication Wastage and Hospital Admissions
}

\author{
Nikhil Khandelwal, PhD, BPharma; Ian Duncan, FSA, FIA, FCIA, MAAA a; Tamim Ahmed, PhD, MBA'; \\ Elan Rubinstein, MPH, PharmD ${ }^{\mathrm{b}}$; and Cheryl Pegus, MD, MPH; Deerfield, Illinois; Farmington, Connecticut; \\ Oak Park, California; and Conshohocken, Pennsylvania
}

\begin{abstract}
Adherence, medication wastage, and reduction in hospital admissions were investigated in a retrospective test-control study design for patients enrolled in the oral chemotherapy cycle management program (CMP), a program that offers clinical support, dose monitoring, and early identification of side effects for patients on select oral chemotherapy. Patients who initiated oral chemotherapy with sorafenib, sunitinib, or erlotinib during June 2008 through December 2009 and who were enrolled in the CMP were included as a test group. Patients who initiated oral chemotherapy with these drugs using Walgreens Specialty Pharmacy during January 2007 through May 2008 and were not part of the CMP were included as control group 1, and patients from a national payor database who initiated therapy with sorafenib, sunitinib, or erlotinib during June 2008 through August 2010 were included as control group 2. Compared with control group 1, patients in the CMP group showed no significant differences with regard to their possession ratios $(P>.05)$, but demonstrated significantly higher persistency rates $(P<.05)$ at the end of 6 months follow-up. For patients in the CMP group who discontinued therapy, approximately $34 \%$ could have experienced reduced wastage had they been on a split medication plan. Patients who are monitored closely and able to identify serious side effects early can avoid complications leading to hospitalizations. The study showed potential savings on drug costs because of a split-fill medication plan, and savings from reduced hospitalization associated with timely identification and management of severe side effects. A clinical program, such as CMP, effectively improves adherence and reduces wastage and hospitalizations for oral chemotherapeutic agents, realizing potential cost savings to both payors and patients. (JNCCN 2012;10:618-625)
\end{abstract}

From aWalgreen Co., Deerfield, Illinois, and 'Solucia Consulting, Farmington, Connecticut.

Submitted March 25, 2011; accepted for publication June 22, 2011.

Drs. Khandelwal, Duncan, and Pegus are employees of Walgreen Co., the funder of this study. Drs. Ahmed and Rubinstein have disclosed that they have no financial interests, arrangements, or affiliations with the manufacturers of any products discussed in this article or their competitors.

Correspondence: Nikhil Khandelwal, PhD, BPharm, 3 Parkway North, Deerfield, IL 60015.

E-mail: Nikhil.Khandelwal@us.astellas.com
Several oral chemotherapies and biologic therapies have been introduced in the past decade for use alone and combined with intravenous chemotherapy. The role of oral chemotherapy agents in cancer management will likely increase. ${ }^{1}$ Oral chemotherapy dosing regimens have been able to "reframe some cancers as chronic diseases requiring chronic therapy." ${ }^{2}$ However, oral agents that are used for liver and renal cancer (i.e., sorafenib), ${ }^{3}$ gastrointestinal stromal tumors (GIST; i.e., sunitinib), ${ }^{4}$ and non-small cell lung (NSCLC) or pancreatic cancers (i.e., erlotinib) ${ }^{5}$ are reported to have the potential for toxicity, ${ }^{6}$ and may pose a significant adherence risk, particularly in the first 30 days of therapy. ${ }^{\text {? }}$ Dose modifications and outright discontinuation of therapy are common responses to these toxicities.

The degree to which a patient follows a treatment regimen as assigned by the health care provider has been described in a variety of ways. The term adherence has been recognized as a major health outcome in today's health care system, and reflects "the extent to which the patient takes medications as prescribed." ${ }^{8}$ Nonadherence is defined as any deviation from physician instructions for the prescribed medications, and exerts a tremendous burden on the health care system in terms of higher costs and poorer health outcomes. Adherence rates for most chronic conditions that use long-term therapies have been shown to be $40 \%$ to $50 \%$. Nonadherence can occur in various forms, such as patient failure to initiate therapy, unclaimed prescriptions, filling the prescription but not consuming the medication as scheduled, or premature discontinuation of medication because of side effects. ${ }^{8}$ Monitoring of adherence is crucial for health care providers, including physicians, because it helps them determine treatment effectiveness, toxicity, and safety. Adherence can be assessed using a variety of direct and indirect methods. 
Oral Chemotherapy Program

Oral chemotherapy is subject to several factors that can impact adherence, and hence total health care costs, including 1) patient self-administration and medication monitoring; 2 ) patients' inability to read, understand, or follow complex instructions for self-administration ${ }^{9}$; 3) compliance risk, which may reflect inadequate adherence, overadherence, or reduced persistency $\left.{ }^{10} ; 4\right)$ inadequate identification and reporting of, and response to, adverse events; 5) drug interactions with food and other medications; and 6) apparent nonresponsiveness to a drug regimen, which may also result in chemotherapy resistance. Apart from these reasons, the complexity of oral chemotherapy regimens adds further challenges to adherence because patients do not always understand the impact of missed doses and may misunderstand directions, which could lead to either underdosing or overdosing.

Limited research is published on adherence to oral anticancer regimens and its relationship to patient outcomes. A study by McCowan et al. ${ }^{11}$ found that only $50 \%$ of women in a cohort completed the 5 -year course of tamoxifen treatment. A recent survey of managed care organizations showed that approximately $45 \%$ of oral chemotherapy is dispensed through retail pharmacy channels, 31\% through specialty pharmacies, $16 \%$ through mail order, and only $7 \%$ through a physician's office. ${ }^{12}$ Because oral chemotherapeutic agents are often covered under the pharmacy benefit plans for individuals who have insurance coverage, they are commonly dispensed through pharmacy channels. Their significant potential toxicity, adherence concerns, high patient self-management requirements, and high costs together suggest the need for targeted pharmacy care management programs to monitor and facilitate appropriate medication use.

Walgreens Oral Chemotherapy Cycle Management Program (CMP) is an evidence-based clinical program that provides clinical management and support to patients who receive sorafenib, sunitinib, or erlotinib for the treatment of liver or kidney cancers; GIST; or NSCLC and pancreatic cancers, respectively. ${ }^{13}$ The CMP team consists of oncology nurses and pharmacists, and provides clinical management through education, monitoring, and counseling of patients via telephone at predetermined intervals. Patients are contacted on day 10 and 20 during the first month of therapy, and monthly thereafter to ensure that they have initiated the prescribed therapy, that any side effects are recorded and graded, and that appropriate management steps are taken. Additionally, an oncology trained pharmacist or nurse conducts a midcycle assessment to determine whether the patient is tolerating the therapy. The midcycle assessments are drug-specific and assess for drug-specific side effects, which are graded according to the National Cancer Institute toxicity criteria. ${ }^{14}$ A drug-specific oncology clinical algorithm crafted and standardized by Walgreens Oncology Advisory Board is used to guide the CMP team to not only ask the patients appropriate questions regarding their therapy and any side effects they may be experiencing, but also identify areas in which appropriate teaching can be provided. Patient education is provided according to the FDA-approved patient information sheets.

If grade 2 or 3 adverse events are noted, the prescribing physician is contacted and a report is faxed, detailing the findings of Walgreens' clinicians along with the dates the patient started/stopped therapy, adherence issues, and side effects. The report requests that the prescribing physician consider this new information and then contact Walgreens regarding any revisions to the oral chemotherapy regimen or use of supportive care drugs that have been indicated. Patients are also provided with a toll-free number at which they can reach a pharmacist $24 / 7$ to discuss any questions regarding their chemotherapy or any side effect issues they may be experiencing.

In an effort to minimize drug waste that may result from therapy modifications, participating plan sponsors may instruct Walgreens Specialty Pharmacy to initially dispense a partial supply of the patient's first month's medication (i.e., "split-fill"), followed by the balance of the month's supply if the patient tolerates the medication and does not experience any serious (i.e., grade 2 or 3 ) adverse events. If serious adverse events, intolerability, therapy ineffectiveness, or patient death are identified at midcycle assessment, the remainder of the month's supply is not dispensed.

The overall objective of the CMP is to address factors that contribute to nonadherence, improve quality and satisfaction of both the patient and provider, and impact cost and wastage. This is achieved through 1) increasing patient understanding and awareness of the treatment regimen and importance 
Khandelwal et al.

of adherence; 2) identifying side effects and complications from medications, which, with appropriate intervention, can reduce complications and adverse events; 3) communicating regularly with patients and health care providers and informing them about wastage prevention from midcycle changes in dosage and discontinuation of therapy; and 4) allowing monthly medications to be shipped as a split-fill, thereby enabling health plans to control potential wastage.

\section{Study Hypotheses}

This study evaluates the impact of the CMP on the key outcomes of quality and cost of care. These outcomes are assessed through measures of adherence, persistency, wastage, and adverse side effects. The 3 hypotheses tested in this study are: 1) patients who participated in the CMP are more likely to have higher adherence (i.e., medication possession ratio [MPR]) and persistency than those in the control group; 2) participation in the split-fill program of the CMP reduces medication wastage; and 3) CMP participants have a lower incidence of drug-associated adverse events, resulting in fewer hospital admissions than the control groups.

\section{Methods}

The clinical use and cost experience of patients in the CMP were compared with those of different control groups (depending on the specific hypothesis).

\section{Study Population}

De-identified data from 3 sources were used for this study.

- The intervention group (CMP group) consisted of approximately 899 patients receiving sorafenib, sunitinib, or erlotinib who were managed as part of the CMP program at Walgreens. The study was restricted to include patients

1. Whose first fill date or index date for any of the 3 drugs (sorafenib, sunitinib, or erlotinib) was between June 2008 and December 2009,

2. Who were new to therapy (with no prescription history for study drugs during the prior 6 months), and

3. For whom survey assessment data were available.
From a total of 1740 patients who initiated their sorafenib, sunitinib, or erlotinib therapy and were enrolled in the CMP between June 2008 and December 2009, 1341 had dispense data, of whom 899 had assessment data available. None of the patients included in the study were part of clinical trials.

- Control group 1 consists of patients receiving one of the identified oral chemotherapy drugs from Walgreens between January 2007 and May 2008 (before CMP implementation) and who completed an assessment survey. From a total of 1153 patients who were identified as taking sorafenib, sunitinib, or erlotinib, 876 patients were identified as being new to therapy, of whom 351 had survey assessment information available.

- Control group 2 was derived from a national payor claims data set (Solucia Consulting, Farmington, CT), containing millions of lives from commercial payor and employer data over several years. New-to-therapy patients with continuous enrollment between June 2008 and August 2010 and who had taken any of the 3 drugs were identified. A total of 622 patients were included in this group. This group was specifically used in testing hypothesis 3 .

Hypothesis 1: Patients in the CMP and control group 1 cohorts were matched using diagnosis and treatment data, and then the propensity mix adjustment method was applied to control for factors such as age, gender, drug, and cancer types (e.g., liver, lung, kidney, pancreatic, GIST). Although the method controlled for drug type, it did not control for cancer stage, first-line versus second-line treatment, or single-agent versus combination therapy. Adherence for each group was assessed using 2 metrics: MPR and persistency rates. The MPR was computed as:

$\left(\mathrm{MPR}_{\mathrm{T}}=\sum\right.$ actual days' supply $\left.\mathrm{t}_{\mathrm{t}}\right) /\left(\sum\right.$ members $_{\mathrm{t}}$ $\times 30)$,

where $t=1$ and MPRT is the measure of medication possession at the end of the Tth month after inception of treatment (index date).

For the purpose of this study, time was limited to 6 months. The denominator includes all identified members who initiated treatment with study drugs in the first month, less those members who died in the first month ( $t-1)$. If a member discontinues in the first 
or subsequent months for reasons other than death, that member is retained in the denominator. Each member's days' supply is tracked in the numerator in each month to provide the medication possession at the end of the last month after inception to treatment.

Persistency rate, or percentage of patients who remain on treatment each month, was also calculated based on all members active at the end of the sixth month. Persistency rate is defined as:

Persistency $_{t}=$ Members active in month $/$

Members active in month $\mathrm{T}_{0}$,

where $T_{0}$ is the number of members on oral chemotherapy in the beginning month.

For both adherence measures, statistical differences across the CMP and control group 1 cohorts were determined by applying the $\chi^{2}$ with the homogeneity of proportion test, and the significance was determined at the $\alpha=0.05$ value.

Hypothesis 2: Patients with cancer conditions may die or discontinue therapy without completing the full treatment cycles (typically 5-7 cycles of 28-30 days each, depending on the medication). ${ }^{15,16}$ Both oral and parenteral chemotherapeutic agents have a narrow therapeutic index, which may lead to an increased risk for side effects. ${ }^{17}$ Moreover, patients who experience serious side effects from treatment may discontinue their medications any time during their treatment cycle, potentially leading to wastage. Through linking assessment data to prescription data in the CMP group, the authors determined the relationship between treatment end date and last medication fill date for the study drugs. Payors who participate in the split-fill program agree to split the supply of a month's medication into 16-day and 14-day batches. When a patient who is not in the split-fill program discontinues therapy midmonth, the remainder of a 30-day supply held by that patient is wasted. However, with the split-fill program, reduction in medication wastage could be either

- 15 days, for which discontinuation occurs anytime between days 1 through 16 of the monthly cycle, or

- Zero days, for which discontinuation occurs anytime between days 17 through 30 of the monthly cycle.

Hypothesis 3: The CMP intervenes early to identify drug-related adverse reactions and events, which may reduce use of inpatient services, such as hospitalizations. The authors hypothesize that patients in the CMP are less likely to experience severe side effects, and will thus experience fewer hospital admissions. The likelihood of hospital admissions related to the study drug use that were incurred during the evaluation period were compared between the CMP and both control groups. Whether the hospitalizations were from an adverse reaction to the drug was difficult to determine from the patient assessment data, because these details were often missing from the survey information. Although the survey data provided information such as whether the patient had any adverse reactions to a given drug in the prior month, the reason for the hospitalization was not collected. Monthly records of hospital admissions extracted from patient assessment data were used for the CMP versus control group 1 comparison, whereas claims-based experience was used for comparing CMP versus control group 2. This hypothesis was tested using a linear mixed regression model that captures both fixed and random effects of the observations. Evidence of hospital admission was used as a dependent variable, whereas independent variables included factors such as age, gender, drug type, clinical attributes (i.e., cancer types), side effects, treatment time, and an intervention variable identifying CMP participation.

\section{Results}

\section{Adherence}

MPR was derived for a total of 377 patients each in the CMP and control group 1 cohorts. Table 1 summarizes adherence rates for the 2 groups. Although month-to-month variations in the MPR was observed across the 2 groups, at the end of 6 months the MPR for the CMP group was found to be slightly higher than the control group 1 (44.8\% vs. $41.5 \%)$. However, the difference was not found to be statistically significant $(P>.05)$.

Table 2 compares persistency rates between CMP and control group 1. Adjusting for member-specific demographics and clinical and side effect profiles, results showed that, starting from month 3, patients in the CMP group had significantly higher persistency rates compared with those in control group 1. Additionally, at the end of 6 months, a significantly higher percentage of patients in the CMP group persisted within their treatment cycles compared with control group $1(23.8 \%$ vs. $7.8 \% ; P<.05)$. 


\section{Split Medication Programs: Impact on Medication Wastage}

In the CMP, if authorized by the payor, each treatment cycle of sorafenib, sunitinib, or erlotinib is supplied as a first-16-day batch followed by a 12- to 14day batch, depending on the medication. However, not all payors provided this authorization. Table 3 summarizes potential reduction in medication wastage across the 3 drugs by month for CMP patients who discontinued therapy. Over the entire study period, a total of 278 of 823 patients $(33.8 \%)$ could have been prevented from wasting medications through implementation of a split-fill medication program. Based on the prevailing average wholesale price of the drug, total potential savings were estimated at $\$ 768,850$, or $\$ 2765.65$ per patient eligible for split-fill.

Patients may discontinue medications for several reasons. Table 4 summarizes potential savings from reduced wastage for discontinuation reasons. Patient death was reported as the most common discontinuation reason of those identified, contributing approximately $21.9 \%$ of potential wastage, followed by physician decision $(10.1 \%)$, therapy ineffective $(9.7 \%)$, switched medication $(9.0 \%)$, and side effects (7.2\%). A large number of discontinuations were recorded as "unknown reason," preventing further analysis. The average potential direct savings that could result from avoiding medication wastage was found to be $\$ 934.20$ per patient who discontinued use.

\section{Side Effects and Adverse Events}

Patients who usually receive oral chemotherapeutic agents such as sorafenib, sunitinib, or erlotinib typically have late-stage cancers. Because these agents are taken by patients themselves without any oversight from the physician, occurrences of serious side effects along with other possible reasons could lead into additional health care resource use, such as emergency room visits or even hospitalizations. Evidence shows that patients who are on oral chemotherapy and experience serious side effects are likely to have emergency room visits or hospitalization. A study conducted by Dana-Farber Cancer Institute showed that patients on oral chemotherapy were more likely to have hospitalizations for side effects related to oral chemotherapeutic agents and incur additional health care costs compared with those not being treated with these agents. ${ }^{18}$ This study determined the probability of incurring hospitalizations

\begin{tabular}{|llll|}
\hline Table 1 & $\begin{array}{c}\text { Comparison of Adherence Rate } \\
\text { (Mean MPR) Between CMP and } \\
\text { Control Group 1 }\end{array}$ & $\begin{array}{l}\text { Pa CMP vs. } \\
\text { Control 1 }\end{array}$ \\
\hline $\begin{array}{l}\text { Mean MPR by } \\
\text { Month }\end{array}$ & CMP & Control 1 & \\
\hline $\begin{array}{l}\text { Total No. of } \\
\text { Patients }\end{array}$ & 377 & 377 & .8792 \\
Month 1 & $98.8 \%$ & $98.9 \%$ & .4758 \\
Month 2 & $72.8 \%$ & $75.2 \%$ & .8491 \\
Month 3 & $64.0 \%$ & $64.7 \%$ & .3367 \\
Month 4 & $58.0 \%$ & $54.3 \%$ & .4921 \\
Month 5 & $50.5 \%$ & $47.8 \%$ & .4016 \\
Month 6 & $44.8 \%$ & $41.5 \%$ & \\
\hline
\end{tabular}

Abbreviations: CMP, cycle management program; MPR, medication possession ratio.

${ }^{a} P$ values are based on $\chi^{2}$ scores.

for patients who were closely monitored for their side effects and therefore were managed appropriately versus those who did not have any oversight from their providers regarding side effect management.

To estimate the impact of the CMP program on the likelihood of hospital admission, the authors tested several linear mixed regression models using unmatched and matched patient samples. Reductions in hospitalization probabilities for all models are shown in Table 5, along with estimated savings from the lower incidence of admissions in the CMP population. Models 1 and 3 represent unmatched patient group comparisons, whereas models 2 and 4 show comparisons for matched patient groups. In all models, hospital admission reductions are found to be significant, from a low of

\section{Table 2 Comparison of Persistency Rates} Between CMP and Control Group 1

\begin{tabular}{|llll|}
$\begin{array}{l}\text { Persistency Rates } \\
\text { by Month }\end{array}$ & CMP & Control 1 & $\begin{array}{l}\boldsymbol{P}^{\mathrm{a}}<\text { CMP vs. } \\
\text { Control 1 }\end{array}$ \\
\hline $\begin{array}{l}\text { Total No. of } \\
\text { Patients }\end{array}$ & 541 & 541 & \\
Month 1 & $100.0 \%$ & $100.0 \%$ & 1.000 \\
Month 2 & $60.1 \%$ & $53.2 \%$ & .0879 \\
Month 3 & $51.2 \%$ & $34.0 \%$ & .0003 \\
Month 4 & $38.3 \%$ & $20.7 \%$ & .0013 \\
Month 5 & $31.2 \%$ & $12.2 \%$ & .0027 \\
Month 6 & $23.8 \%$ & $7.8 \%$ & .0234 \\
\hline
\end{tabular}

Abbreviation: CMP, cycle management program. ${ }^{\text {a }} P$ values are based on $\chi^{2}$ scores. 
Oral Chemotherapy Program

\begin{tabular}{|c|c|c|c|c|c|}
\hline Discontinuation Month & $\begin{array}{l}\text { All } \\
(\mathrm{N})\end{array}$ & $\begin{array}{l}\text { Wastage } \\
\text { (N) }\end{array}$ & $\begin{array}{l}\text { Wastage } \\
(\%)\end{array}$ & $\begin{array}{l}\text { Potential } \\
\text { Savings }\end{array}$ & $\begin{array}{l}\text { Average Savings Per Patient } \\
\text { With Wastage }\end{array}$ \\
\hline Month 1 & 261 & 20 & $7.7 \%$ & $\$ 53,167$ & $\$ 2658.34$ \\
\hline Month 2 & 219 & 103 & $47.0 \%$ & $\$ 288,387$ & $\$ 2799.87$ \\
\hline Month 3 & 134 & 62 & $46.3 \%$ & $\$ 169,758$ & $\$ 2738.04$ \\
\hline Month 4 or later & 209 & 93 & $44.5 \%$ & $\$ 257,538$ & $\$ 2769.23$ \\
\hline Subtotal & 823 & 278 & $33.8 \%$ & $\$ 768,850$ & $\$ 2765.65$ \\
\hline
\end{tabular}

${ }^{a}$ Based on average wholesale price.

2.9\% (for model 2 ) to a high of $5.3 \%$ for model 4 . The overall reduction derived from the average of hospital reductions across all 4 models was found to be $3.6 \%$.

Through linking hospital admissions to the drug type, the authors estimated costs associated with each type of admission and applied those average admission costs to derive potential cost savings across each model. Estimated savings ranged from $\$ 369.38$ to $\$ 672.96$ per patient, as shown in Table 5 , and the overall estimated savings was found to be $\$ 452.21$ per patient, which is the indirect savings from reduced hospitalizations.

\section{Conclusions}

When side effects from oral chemotherapy are not managed appropriately, patients are more likely to stop taking these drugs as prescribed or discontinue their therapy altogether, affecting their medication possession or their persistency level. Because patients in the
CMP are closely monitored by trained clinicians, they are more likely to be titrated for dosage changes or therapy switches, and, with prescriber authorization, therapy may be suspended until side effects subside. MPR and persistency rates were compared between patients managed through the CMP and those in the control groups. MPR and persistency metric evaluation reveals 2 different stories. MPR measures the possession ratio, or the rate of patients filling prescriptions. However, it does not accurately measure how much drug is actually consumed. Persistency rates, however, could be a more relevant patient adherence measure, especially in cancer conditions, because it combines both patient survival and completion of treatment into a single metric.

No significant differences were seen between the $\mathrm{CMP}$ and control group 1 cohorts regarding their possession ratios (MPR), but adherence through persistency rates did indicate significantly higher persistency rates for the CMP group than control group 1 .

The split-fill medication program reduces wastage in members who discontinue midcycle. Analy-

\section{Table 4 Wastage Savings From Discontinuationa}

\begin{tabular}{|lccccl|}
\hline Discontinuation Reason & $\begin{array}{c}\text { All } \\
(\mathbf{N})\end{array}$ & $\begin{array}{c}\text { Wastage } \\
(\mathbf{N})\end{array}$ & $\begin{array}{c}\text { Wastage } \\
(\%)\end{array}$ & $\begin{array}{l}\text { Potential Savings } \\
\text { Discontinued Patient }\end{array}$ \\
\hline Deceased & 172 & 61 & $21.9 \%$ & $\$ 161,114$ & $\$ 2641.22$ \\
Physician decision & 80 & 28 & $10.1 \%$ & $\$ 73,598$ & $\$ 2628.49$ \\
Therapy ineffective & 85 & 27 & $9.7 \%$ & $\$ 66,621$ & $\$ 2467.46$ \\
Switched medication & 53 & 25 & $9.0 \%$ & $\$ 94,404$ & $\$ 3776.15$ \\
Side effects & 94 & 20 & $7.2 \%$ & $\$ 48,413$ & $\$ 2420.66$ \\
Unable to contact & 65 & 11 & $4.0 \%$ & $\$ 33,625$ & $\$ 3056.79$ \\
Unknown & 130 & 54 & $19.4 \%$ & $\$ 151,633$ & $\$ 2808.01$ \\
Others & 178 & 52 & $18.7 \%$ & $\$ 139,442$ & $\$ 2681.58$ \\
Total & 823 & 278 & $100.0 \%$ & $\$ 768,850$ & $\$ 2765.65$ \\
Savings per eligible member & & & & & $\$ 934.20$ \\
\hline
\end{tabular}

aBased on average wholesale price. 
Khandelwal et al.

sis showed that for the patients who discontinued therapy, approximately $34 \%$ could have experienced reduced wastage had they been on a split-fill medication plan resulting in direct savings of approximately $\$ 934.20$ per patient who discontinued use. However, split-fill medication programs are not always available under employers' health benefit plans.

The impact of the CMP program on hospitalizations was also analyzed. Patients who can manage side effects successfully, or are monitored closely and able to identify serious side effects early, may also avoid complications that lead to hospitalizations. We tested various models, matching patients in various ways between the CMP and control groups, and found reductions in likelihood of hospitalization compared to the control groups.

The study showed potential savings from a splitfill medication plan and savings from reduced hospitalization associated with timely identification and management of severe side effects for the 3 oral chemotherapy drugs. Findings showed approximately $3.6 \%$ reductions in the likelihood of hospitalization for the CMP group compared with the control group, thereby providing an additional indirect savings of approximately $\$ 452.21$ per patient. Overall, the combined savings resulting from reduced wastage and hospital admissions, expressed on a per-patient basis, was found to be $\$ 1386.41$.

\begin{tabular}{|c|c|c|c|}
\hline Models & $\begin{array}{l}\text { Admission } \\
\text { Reductions } \\
(P)\end{array}$ & $\begin{array}{l}\text { Cost Per } \\
\text { Hospital } \\
\text { Admission }\end{array}$ & Savings \\
\hline $\begin{array}{l}\text { Model 1: CMP vs. } \\
\text { control } 1\end{array}$ & $\begin{array}{l}-0.0332^{\mathrm{a}} \\
(.0440)\end{array}$ & $\$ 12,403.03$ & $\$ 411.78$ \\
\hline $\begin{array}{l}\text { Model 2: CMP vs. } \\
\text { control 1, matched }\end{array}$ & $\begin{array}{l}-0.0294^{b} \\
(.0880)\end{array}$ & $\$ 12,563.97$ & $\$ 369.38$ \\
\hline $\begin{array}{l}\text { Model 3: CMP vs. } \\
\text { control } 2\end{array}$ & $\begin{array}{l}-0.03200^{a} \\
(.0002)\end{array}$ & $\$ 13,187.39$ & $\$ 417.98$ \\
\hline $\begin{array}{l}\text { Model 4: CMP vs. } \\
\text { control 2, matched }\end{array}$ & $\begin{array}{l}-0.0534^{\mathrm{a}} \\
(.0030)\end{array}$ & $\$ 12,599.82$ & $\$ 672.96$ \\
\hline All & $-3.6 \%$ & $\$ 12,736.81$ & $\$ 452.21$ \\
\hline
\end{tabular}

Abbreviation: CMP, cycle management program.

${ }^{\text {a }} P<.05$.

${ }^{\mathrm{b}} P<.1000$.

\section{Discussion}

Oral chemotherapy has gained popularity with patients over the past decade as a convenient approach for certain cancer treatments. However, the rapid increase in the prescribing of oral chemotherapy creates therapy management challenges; although oral chemotherapy may reduce the number of office visits, it also transfers decision-making and monitoring responsibility to patients and/or their caregivers. Patients must understand how to detect and deal with side effects that may accompany particular oral chemotherapies. The CMP is designed to support patients taking self-administered oral chemotherapy, and educate and closely monitor those taking particular drugs. Repeated surveys are performed to understand side effects, adherence, and dosage titration to maximize the impact of oral chemotherapy.

Patients on oral oncology medications who are not managed appropriately are more likely to experience unmanaged side effects. Trained clinicians in the oral chemotherapy CMP offer clinical support to patients and provide dose monitoring and early identification of side effects. The CMP adds value by

- reducing medication wastage that results from discontinuation, and

- reducing hospitalizations through timely management of side effects, resulting in a potential indirect savings.

Great potential exists for health plans and payors to improve quality and patient satisfaction and achieve significant cost savings through participating in programs, such as CMP, that actively manage patients taking oral chemotherapy.

\section{References}

1. Schwartz RN, Eng KJ, Frieze DA, et al. NCCN Task Force report: specialty pharmacy. J Natl Compr Canc Netw 2010;8(Supp 4):S112.

2. Weingart SN, Brown E, Bach PB, et al. NCCN Task Force report: oral chemotherapy. J Natl Compr Canc Netw 2008;6(Supp 3):S114 .

3. Vanelli M, Pedan A, Liu N, et al. The role of patient inexperience in medication discontinuation: a retrospective analysis of medication non persistence in seven chronic illnesses. Clin Ther 2009;31:2628-2652.

4. Nexavar (sutent) [package insert]. Wayne, NJ: Bayer HealthCare Pharmaceuticals Inc.; 2010.

5. Sutent (sunitinib) [package insert]. New York, NY: Pfizer Inc.; 2010. 
Oral Chemotherapy Program

6. Cancer Therapy Evaluation Program. CTEP, NCI Guidelines: Adverse Event Reporting Requirements. Effective January 1, 2005. National Cancer Institute Web site. Available at: http://ctep. cancer.gov/protocolDevelopment/electronic_applications/docs/ newadverse_2006.pdf. Accessed March 13, 2012.

7. Tarceva (erlotinib) [package insert]. Farmingdale, NY: OSI Pharmaceuticals, LLC; 2010.

8. Berger BA, Krueger KP, Felkey BG. The pharmacist's role in treatment adherence. Part 1: extent of the problem. US Pharmacist 2004;29:50-54.

9. Wolf MS, Davis TC, Shrank W, et al. To err is human: patient misinterpretations of prescription drug label instructions. Patient Educ Couns 2007;67:293-300.

10. Egorin MJ. Promises and pitfalls of oral cancer chemotherapy. Clin Adv Hematol Oncol 2009;7:S8-10.

11. McCowan C, Shearer J, Donnan PT, et al. Cohort study examining tamoxifen adherence and its relationship to mortality in women with breast cancer. Br J Cancer 2008;99:1763-1768.

12. Blansett L. Changing reimbursement policies and trends, presentation to association of community cancer centers. 2009. Available at: www.amcp.org/WorkArea/DownloadAsset. aspx?id=11843. Accessed March 20, 2012.

13. Outlook: state of the industry report 2009. Walgreens Specialty Pharmacy. Available at: http://www.walgreenshealth.com/pdf/ newsletterreport/SOIReport2009.pdf. Accessed March 13, 2012.

14. Cancer Therapy Evaluation Program. Common Toxicity Criteria Manual. Version 2.0. June 1, 1999. National Cancer Institute Web site. Available at: http://ctep.cancer.gov/protocoldevelopment/ electronic_applications/docs/ctcmanual_v4_10-4-99.pdf. Assessed May 20, 2011.

15. Eisen T, Oudard S, Gravis G et al. Sorafenib for older patients with renal cell carcinoma: subset analysis from a randomized trial. J Natl Cancer Inst 2008;100:1454-1463.

16. Motzer RJ, Agarwal N, Beard C, et al. NCCN Clinical Practice Guidelines in Oncology: Kidney Cancer. Version 2, 2012. Available at: NCCN.org. Accessed March 24, 2011.

17. Moody M, Jackowski J. Are patients on oral chemotherapy in your practice setting safe? Clin J Oncol Nurs 2010;14:339-346.

18. Hassett MJ, O'Malley AJ, Pakes JR, et al. Frequency and cost of chemotherapy-related serious adverse effects in a population sample of women with breast cancer. J Natl Cancer Inst 2006;98:11081117. 